\title{
Risk assessment modelling of fecal shedding caused by extended-spectrum cephalosporin-resistant Escherichia coli transmitted through waste milk fed to dairy pre-weaned calves
}

\author{
Babafela B. Awosile*1 and Ben A. Smith† \\ *Department of Health Management, Atlantic Veterinary College, University of Prince Edward Island, Charlottetown, PE, C1A 4P3, Canada \\ †Public Health Risk Sciences Division, Public Health Agency of Canada, 160 Research Lane, Guelph, Ontario N1G 5B2, Canada
}

\begin{abstract}
Waste milk feeding is a common practice in dairy operations. Regardless of the benefits of this practice to the dairy farmers, concerns from the potential dissemination of antimicrobial-resistant bacteria through the gut and subsequent shedding by calves into the environment are increasing. In this study, we employed Monte Carlo simulation to assess the risk of shedding extended-spectrum cephalosporin-resistant Escherichia coli (ESC-R E. coli) caused by waste milk feeding in pre-weaned calves using an exponential dose-response model fit to data for E. coli O157:H7 in cattle. Data from pertinent studies were included in our model to predict the risk of shedding. The median (5th and 95th percentiles) for the daily risk of shedding ESC-R $E$. coli by calves fed only contaminated waste milk was predicted to be $2.9 \times 10^{-3}\left(2.1 \times 10^{-3}, 3.7 \times 10^{-3}\right)$, representing a median daily risk of 29 out of 10,000 calves shedding ESC-R E. coli due to exclusive feeding of waste milk containing ESC-R E. coli. This median value was reduced by $94 \%$ when accounting for the proportion of waste milk that does not contain ESC-R $E$. coli. The overall risk of shedding ESC-R E. coli through the pre-weaning period for farms that feed waste milk to calves was $5.7 \times 10^{-3}\left(2.4 \times 10^{-3}, 1.1 \times 10^{-2}\right)$, representing 57 out of 10,000 calves. When accounting for the proportion of farms that do not feed waste milk, the pre-weaning period risk was reduced by $23 \%$. By varying the prevalence of ESC-R E. coli in waste milk using values of $3,1.5$, and $1 \%$, the daily risk of shedding decreased by factors of 50,65 , and $82 \%$, respectively, which supports the reduction of contamination or discontinuation of feeding waste milk containing ESC-R E. coli as major mitigation measures to reduce the risk of shedding caused by ingestion of resistant bacteria. It is
\end{abstract}

Received May 19, 2017.

Accepted August 18, 2017.

${ }^{1}$ Corresponding author: bawosile@upei.ca anticipated that the effects of antimicrobial residues in waste milk, which was not considered herein due to lack of data, would further increase risks. Although waste milk feeding to calves may be economically beneficial to the dairy farmers, there exists the risk of dissemination of ESC-resistant bacteria into the environment.

Key words: quantitative microbial risk assessment, antimicrobial resistance, AMR, Monte Carlo simulation, mathematical model

\section{INTRODUCTION}

Antimicrobial resistance attributed to veterinary use is a concern for both public health and food animal production systems; this is as a result of increasing levels of resistance in foodborne zoonotic bacteria and clinical pathogens (van den Bogaard and Stobberingh, 2000). In dairy production in some parts of the world, antimicrobials are commonly administered to dairy cows as an intramammary infusion or injectable for the treatment of mastitis and, during the dry-off, either as blanket or selective treatment for the prevention or treatment of mastitis (McEwen and Fedorka-Cray, 2002; Saini et al., 2012). Feeding milk from these cows to dairy calves is a common practice on many dairy farms (Duse et al., 2013). Such milk may contain multidrug-resistant bacteria (Wray et al., 1990; Brunton et al., 2012) as well as antimicrobial residues (Pereira et al., 2014a), which may facilitate horizontal transfer of resistant genes and selection pressure on the calves' gastrointestinal bacteria, respectively. Sub-therapeutic antimicrobial exposure through waste milk feeding practices may explain to some extent the high prevalence of multi-drug-resistant bacteria such as Escherichia coli in pre-weaned calves (Aust et al., 2012). Economic considerations and convenience (Godden et al., 2005; Abb-Schwedler et al., 2014), environmental protection, and difficulty in disposal (Brunton et al., 2012) have been reported as some of the reasons for waste milk feeding in dairy farms. 
Extended-spectrum cephalosporins (ESC) such as ceftiofur and cefquinome are commonly used in dairy production as intramammary preparation for treatment purposes against mastitis (Saini et al., 2012; Brunton et al., 2014; Randall et al., 2014). Therapeutic effectiveness and short withdrawal times make this class of antimicrobials increasingly important in dairy farming (Snow et al., 2012). However, emergence of antimicrobial resistance due to selection pressure by production of extended-spectrum $\beta$-lactamases (ESBL) and other $\beta$-lactamases has been documented (Daniels et al., 2009; Cormier et al., 2015). Different studies have reported on the detection of cephalosporin residues (Brunton et al., 2014; Pereira et al., 2014a; Randall et al., 2014), ESC-resistant (ESC-R) bacteria, and $\beta$-lactamase resistance genes in waste milk (Brunton et al., 2014; Randall et al., 2014) as well as relationships between feeding waste milk containing cephalosporin residues to calves and fecal shedding of ESBL-producing E. coli (Pereira et al., 2014b). A recent report by the European Food Safety Authority examined the risk from feeding waste milk containing antimicrobial residues to calves using a qualitative approach and concluded that consumption of waste milk containing antimicrobial residues leads to subsequent shedding of resistant bacteria, and that this process occurs in practice [EFSA Panel on Biological Hazards (BIOHAZ) et al., 2017].

Thus far, limited information is available establishing the relationship between feeding waste milk containing resistant bacteria and fecal shedding in calves. Quantitative microbial risk assessment (QMRA) has rarely been applied to study this relationship. Available studies include a previous risk assessment in the United Kingdom that reported that $60 \%$ of calves in 5,000 simulated farms were shedding ESC-R E. coli caused by antibiotic residues and ESBL E. coli in waste milk (Simmons et al., 2015). Quantitative microbial risk assessment is a scientific tool that quantifies the level of exposure and the subsequent risk due to a specific bacterial pathogen of concern (Lammerding and Fazil, 2000; Snary, 2004). This assessment involves 4 science-based investigation steps: hazard identification, exposure assessment, hazard characterization, and risk characterization (Lammerding and Fazil, 2000). The output of the risk assessment process represents the probability and effect of exposure to a hazard or adverse event. The QMRA process is useful to direct risk management efforts by examining the effects of model inputs on results through sensitivity analysis, modeling the effects of interventions, and identifying data gaps for further research efforts. The aim of our study was to adopt the QMRA approach to quantify the general risk of fecal shedding of ESC-R E. coli from feeding of waste milk containing ESC-R E. coli to pre-weaned calves using Monte Carlo simulation.

\section{MATERIALS AND METHODS}

\section{Risk Assessment Model}

The scientific literature was searched in Google Scholar (https://scholar.google.ca/) and PubMed (https:// www.ncbi.nlm.nih.gov/pubmed/) databases using the key words of each of the input parameter definitions in Table 1, and data were extracted from the relevant articles. The risk assessment was not tailored to any single region (e.g., country, continent), resulting in the generation of a generic model (i.e., not geographically specific). Whenever possible, input data used in the model were represented by distributions rather than discrete point estimates. The variability for prevalence data inputs was incorporated using $\beta$ distributions, where possible. However, where only few observations were available for input variables, uniform or triangular distributions were used to account for the uncertainty associated with the input data. Using the software @RISK (Palisade Corporation, New York, NY) as an add-on package in Microsoft Excel 2010 (Microsoft Corp., Redmond, WA), Monte Carlo simulation using Latin hypercube sampling was performed using 10,000 iterations. The model outputs a probabilistic distribution of the risk of fecal shedding of ESC-R E. coli due to waste milk feeding in dairy calves. Sensitivity analysis was also carried out to explore different scenarios and their effect on the risk of shedding using Spearman rank correlation coefficients.

\section{Hazard Identification}

Waste milk feeding is a common practice widely used in dairy operations [EFSA Panel on Biological Hazards (BIOHAZ) et al., 2017]. Regardless of the benefits of this practice to dairy farmers, concerns from the potential for development of antimicrobial resistance and subsequent shedding are increasing due to (1) antimicrobial residues and selective pressure on gastrointestinal microflora of calves, and (2) transmission as well as lateral transfer of resistant bacteria within the gastrointestinal tract. Apart from the increased risk of antimicrobial resistance selection among the intestinal microflora due to antimicrobial residues in waste milk, contamination of waste milk with infectious pathogens and multi-drug-resistant bacteria due to pooling of waste milk from different sources has been documented (Wray et al., 1990; Aust et al., 2013). The introduction, multiplication, and establishment of multi-drug- 
resistant bacteria such as $E$. coli within the gastrointestinal microbiota depend on their ability to effectively compete and subdue the normal microbiota (Pereira et al., 2014b). This disruption, due to developing gastrointestinal microflora and an underdeveloped immune system, results in colonization and subsequent shedding of multi-drug resistant bacteria with higher competitive and fitness advantage (Edrington et al., 2012). This is true even in the absence of antimicrobial use.

In studies where antimicrobial resistant bacteria such as E. coli were administered orally (Besser et al., 2001), in milk replacer (Alali et al., 2004) or waste milk (Aust et al., 2013; Brunton et al., 2014) to pre-weaned calves, the proportion of resistant $E$. coli shed was significantly higher in inoculated or waste-milk-fed calves, and shedding continued for several weeks. Cephalosporinresistant bacteria and associated resistance genes in waste milk have been documented for $E$. coli, with an average contamination prevalence of $5.7 \%$ for waste milk contamination with ESC-R E. coli (Randall et al., 2014). Also, increased fecal prevalence of multi-drug resistant $E$. coli has been reported in pre-weaned calves fed waste milk; for example, the proportion of isolates resistant to cefotaxime and cephalothin in calf feces were 3.75 and 1.4 times greater, respectively, in calves fed waste milk versus bulk milk (Aust et al., 2013). For these reasons, ESC-R E. coli was selected as the specific hazard for this assessment.

\section{Exposure Assessment}

Probability of exposure was assessed by the proportion of farms feeding waste milk to calves and the prevalence of ESC-R E. coli in waste milk at feeding. The proportion of farms feeding waste milk to calves varies among studies. A Swedish study reported that $56 \%$ of dairy farms fed waste milk to calves (Duse et al., 2013). Other studies from Austria and Canada have reported prevalence values of $29 \%$ (Klein-Jöbstl et al., 2015 ) and $48.2 \%$ (Vasseur et al., 2010), respectively, whereas proportions as high as 65 to $83 \%$ were reported in the United Kingdom (Brunton et al., 2012; Snow et al., 2012). These reported proportions were used in this assessment by combining the numerical results from each study in a single $\beta$ distribution (Table 1 ). The results reported by Randall et al. (2014) were used to determine the probability of exposure to ESC-R E. coli given waste milk feeding (represented by a $\beta$ distribution with a mean of 5.7\%). Dose was determined based on the average daily quantity of waste milk fed to calves and the concentration of ESC-R E. coli in waste milk. Different studies have reported on the daily frequency and volume of waste milk feeding to calves; calves are fed waste milk 2 times daily with a daily 
quantity ranging from 3.5 to $8 \mathrm{~L} / \mathrm{d}$, for an average of $35 \mathrm{~d}$ through the pre-weaning period (Vasseur et al., 2010; Pereira et al., 2014b). In our model, calves were either fed waste milk for an average of $35 \mathrm{~d}$ during the pre-weaning period, regardless of pre-weaning duration, or not at all. The average concentration of $10 \mathrm{cfu} / \mathrm{mL}$ of ESC-R E. coli in waste milk was used to derive the exposure dose (Randall et al., 2014; Table 1). While we are aware that ingestion of ESC residues in waste milk will influence the shedding of resistant bacteria by calves, a lack of dose-response relationship precluded its consideration in the QMRA.

\section{Hazard Characterization}

Dose-response models characterize the relationship between the ingested dose and the probability of endpoint of interest (i.e., shedding of ESC-R E. coli). There are no known dose-response models for E. coli shedding in calves; most available dose-response models are based on human, rabbit, or pig studies (Pai et al., 1986; Haas et al., 2000; Powell et al., 2000). A dose-response model for $E$. coli shedding in cattle was developed using data from an experimental study in adult cattle. Cray and Moon (1995) studied infection and shedding of $E$. coli $\mathrm{O} 157: \mathrm{H} 7$ in both calves and adult cattle; however, only one dose was studied in the former group, precluding generation of a dose-response relationship based on calf data. Five adult cattle were inoculated with $10^{4}$ cfu, another 5 adult cattle inoculated with $10^{7} \mathrm{cfu}$, and 17 other adult cattle were inoculated with $10^{10} \mathrm{cfu}$ of E. coli O157:H7 strain 308. Magnitude and duration of fecal shedding of E. coli O157:H7 were then assessed; 0,40 , and $100 \%$ of the cattle were positive for infection with E. coli O157:H7 strain 308, respectively. Both $\beta$-Poisson and exponential models were fit to the doseresponse data because they are known to provide good fits to microbial dose-response data (Haas et al., 2000). The parameters of the models were determined by the maximum likelihood method (Haas, 1994), whereas the improvement in fit of the dose-response data between the 2 models was determined using the likelihood ratio test (Haas et al., 2000).

\section{Risk Characterization}

To determine the probability of shedding when exposed to ESC-R E. coli in waste milk, the calculated dose from the exposure assessment was used as an input into the dose-response model. This value was then multiplied by the likelihood of waste milk containing ESC-R E. coli to determine the daily risk of shedding ESC-R E. coli from waste milk feeding (Table 1). The daily risk was then used to compute the pre-weaning period risk for an average of $35 \mathrm{~d}$ of waste milk feeding (Vasseur et al., 2010; Pereira et al., 2014b). Finally, the prevalence of operations that use waste milk as feed was used to determine the overall risk of shedding across all production (considering that some calves are never fed waste milk; Table 1).

\section{RESULTS AND DISCUSSION}

Monte Carlo simulation was used in this study to determine the daily and pre-weaning period risk of subsequent shedding attributed to feeding waste milk containing ESC-R E. coli. The model was simulated to represent exposures of 10,000 calves to ESC-R E. coli in waste milk and the risk is presented in Table 2. The median (5th and 95th percentiles) for the daily risk of shedding ESC-R E. coli by calves fed only contaminated waste milk was predicted to be $2.9 \times 10^{-3}(2.1 \times$ $10^{-3}, 3.7 \times 10^{-3}$ ) representing a median daily risk of 29 out of 10,000 calves shedding ESC-R E. coli due to exclusive feeding of waste milk containing ESC-R E. coli. This value was reduced by $94 \%$ when accounting for the proportion of waste milk that does not contain ESC-R E. coli. The median overall risk of shedding ESC-R E. coli through the pre-weaning period for farms that feed waste milk to calves was $5.7 \times 10^{-3}$, representing 57 out of 10,000 calves over $35 \mathrm{~d}$. When accounting for the proportion of farms that do not feed waste milk, the pre-weaning period risk was reduced by $23 \%$ (Table 2).

Risks associated with waste milk feeding can be mitigated by reducing the prevalence of ESC-R E. coli in waste milk, or limiting the practice of feeding waste milk to calves. Milk feeding replacement and discontinued waste milk feeding practices has been reported to be the most effective measures for the mitigation of risk of shedding of ESC-R E. coli by calves (DEFRA, 2013). In our study, sensitivity analysis using Spearman rank correlation coefficients revealed that prevalence of ESC-R E. coli in waste milk had a strong influence on the risk of shedding $(\mathrm{r}=0.9)$, which suggests this variable to be a key factor to consider in risk mitigation processes. By varying the prevalence of ESC-R E. coli in waste milk using values of $3,1.5$, and $1 \%$, the daily risk of shedding decreased by factors of 50,65 , and $82 \%$, respectively, which further supports the discontinuation of feeding waste milk containing ESC-R E. coli as a major mitigation measure to reduce the risk of shedding caused by ingestion of resistant bacteria. Environmental control measures, prudent use of thirdgeneration cephalosporins (DEFRA, 2013), and onfarm pasteurization of waste milk have been proposed as a means of controlling disease pathogen transmission (Godden et al., 2005). Aust et al. (2013) have reported a drastic reduction in coliform count in waste milk from 
$6.5 \times 10^{2} \mathrm{cfu} / \mathrm{mL}$ to $<10^{1} \mathrm{cfu} / \mathrm{mL}$ following pasteurization.

In this study, a dose-response model was developed to provide an estimate of the relationship between ingestion of ESC-R E. coli and subsequent shedding of ESC-R E. coli by calves. Hazard characterization is a critical step in risk assessment; however, dose-response parameters for analysis are often unavailable or difficult to parameterize. Use of expert judgment, parameters for surrogate pathogens, or parameters obtained via studies with different animals or humans have been used for QMRA (Lammerding and Fazil, 2000). Such assumptions, while often necessary due to a paucity of data, increase the uncertainty associated with risk estimates. In this study, several major assumptions were made to compensate for data gaps in the literature pertaining to the dose-response relationship, namely (1) the dose-response for $E$. coli $\mathrm{O} 157$ was representative of generic E. coli; (2) dose-response data for bacteria with an unknown antimicrobial resistance profile were representative of ESC-R bacteria; and (3) dose-response data based on data from adult cattle were suitable to represent claves. There is a strong possibility the relationship differs in calves compared with adults, considering the higher rate of shedding of resistant bacteria in calves compared with adult cattle (Call et al., 2008). Therefore, it is uncertain to what degree this dose-response model adequately represents the modeled situation; nevertheless, it was based on the most representative data available in the literature. Future experimental studies incorporating use of varying doses of ESC-R E. coli fed in waste milk to calves will generate dose-response data to minimize the aforementioned limitations associated with our dose-response model. As a whole, the lack of dose-response information particular to antimicrobial resistant bacteria is a major data gap that could be addressed by primary research.

We fit both the $\beta$-Poisson and exponential models to dose-response data. From the dose-response analysis, the deviances of both the exponential and $\beta$-Poisson dose-response relationship were nearly identical (varying by $\left.1.2 \times 10^{-6}\right)$. The fit of both models to the doseresponse data was acceptable as evident in the goodness of fit test ( $p \approx 1.0$ for both models), indicating that both models adequately describe the relationship. Best fit model parameters were $\beta$-Poisson: $\alpha=9.47 \times$ $10^{2}, \beta=1.86 \times 10^{10}$; and exponential: $\mathrm{r}=5.10 \times 10^{-8}$. The exponential dose-response model was selected for use in the risk assessment model due to the high $\alpha$ parameter associated with the $\beta$-Poisson fit, indicating an exponential relationship. Therefore, it can be interpreted from the model parameters that ingestion of 1 cell of ESC-R E. coli results in a $5.10 \times 10^{-8}$ probability of fecal shedding of ESC-R E. coli by calves. 
Waste milk feeding to calves may be economically beneficial to dairy farmers; the risk of shedding exists from feeding waste milk to calves. In studies where antimicrobial-resistant bacteria such as E. coli were administered orally (Besser et al., 2001) in milk replacer (Alali et al., 2004) or waste milk (Aust et al., 2013; Brunton et al., 2014) to pre-weaned calves, the proportion of resistant E. coli shed was significantly higher in inoculated or waste milk-fed calves, and shedding continued for several weeks. We incorporated data from different parts of the world including Europe and North America, compared with a previous study (Simmons et al., 2015), which was based only on data collected in the United Kingdom. The risk predictions herein were based only on ESC-R E. coli ingestion and did not consider the effects of antimicrobial residues as well as horizontal transfer of resistance within the guts of calves.

While QMRA provides an objective quantification of risk from exposure to hazards as well as the need for measures to mitigate such risks, it can also help to highlight the data gaps and areas where future research is needed. The existence of limited data contributes to uncertainty in risk assessment models. One major limitation in this study is that within gut horizontal transfer of ESC-resistance and effect of cephalosporin residues were not evaluated due to lack of data. This exclusion likely results in an underestimation of risks. Also, a point estimate of the concentration of ESC-R $E$. coli in waste milk was used in this study due to lack of data. This estimate could be improved with more studies measuring, specifically, the concentration of ESC-R bacteria in waste milk to better characterize the variability in the input parameter. Also, as previously discussed, more dose-response studies are needed on feeding and subsequent shedding of ESC-R E. coli in calves. The indirect risk to humans through veal calves has been suggested (Randall et al., 2014) as these resistant bacteria are transmitted through the food chain; this should be explored using QMRA in future studies.

\section{ACKNOWLEDGMENTS}

We acknowledge the NSERC CREATE-ITRaP scholarship program of Western College of Veterinary Medicine, Saskatoon, Canada, for providing financial support to Babafela Awosile towards the 2-month internship placement with the Public Health Agency of Canada.

\section{REFERENCES}

Abb-Schwedler, K., A. Maeschli, R. Boss, H. U. Graber, A. Steiner, and P. Klocke. 2014. Feeding mastitis milk to organic dairy calves:
Effect on health and performance during suckling and on udder health at first calving. BMC Vet. Res. 10:267. https://doi.org/10 $.1186 / \mathrm{s} 12917-014-0267-7$.

Alali, W. Q., J. M. Sargeant, T. G. Nagaraja, and B. M. DeBey. 2004. Effect of antibiotics in milk replacer on fecal shedding of O157: H7 in calves. J. Anim. Sci. 82:2148-2152.

Aust, V., K. Knappstein, H.-J. Kunz, H. Kaspar, J. Wallmann, and M. Kaske. 2013. Feeding untreated and pasteurized waste milk and bulk milk to calves: Effects on calf performance, health status and antibiotic resistance of faecal bacteria. J. Anim. Physiol. Anim. Nutr. (Berl.) 97:1091-1103. https://doi.org/10.1111/jpn.12019.

Besser, T. E., B. L. Richards, D. H. Rice, and D. D. Hancock. 2001. Escherichia coli O157:H7 infection of calves: Infectious dose and direct contact transmission. Epidemiol. Infect. 127:555-560.

Brunton, L. A., D. Duncan, N. G. Coldham, L. C. Snow, and J. R. Jones. 2012. A survey of antimicrobial usage on dairy farms and waste milk feeding practices in England and Wales. Vet. Rec. 171. https://doi.org/10.1136/vr.100924.

Brunton, L. A., H. E. Reeves, L. C. Snow, and J. R. Jones. 2014. A longitudinal field trial assessing the impact of feeding waste milk containing antibiotic residues on the prevalence of ESBL-producing Escherichia coli in calves. Spec. Issue SVEPM 2014 Support. Decis. Mak. Anim. Health Adv. Multidiscip. Methodol. 2014 Soc. Vet. Epidemiol. Prev. Med. Conf. 117:403-412. https://doi.org/10 .1016/j.prevetmed.2014.08.005.

Call, D. R., M. A. Davis, and A. A. Sawant. 2008. Antimicrobial resistance in beef and dairy cattle production. Anim. Health Res. Rev. 9:159-167. https://doi.org/10.1017/S1466252308001515.

Cormier, A. C., G. Chalmers, T. A. McAllister, S. Cook, R. Zaheer, H. M. Scott, C. Booker, R. Read, and P. Boerlin. 2015. Extended-spectrum-cephalosporin resistance genes in Escherichia coli from beef cattle. Antimicrob. Agents Chemother. 60:1162-1163. https://doi.org/10.1128/AAC.02516-15.

Cray, W. C., and H. W. Moon. 1995. Experimental infection of calves and adult cattle with Escherichia coli O157:H7. Appl. Environ. Microbiol. 61:1586-1590.

Daniels, J. B., D. R. Call, D. Hancock, W. M. Sischo, K. Baker, and T. E. Besser. 2009. Role of ceftiofur in selection and dissemination of blaCMY-2-mediated cephalosporin resistance in Salmonella enterica and commensal Escherichia coli isolates from cattle. Appl. Environ. Microbiol. 75:3648-3655. https://doi.org/10.1128/AEM .02435-08.

DEFRA (Department for Environment, Food and Rural Affairs). 2013. Assessment of the risk of selection of ESBL resistance in calves by feeding waste milk containing antibiotic residues. Evidence project final report OD2031, pages 1-28. http://randd.defra.gov.uk/.

Duse, A., K. P. Waller, U. Emanuelson, H. E. Unnerstad, Y. Persson, and B. Bengtsson. 2013. Farming practices in Sweden related to feeding milk and colostrum from cows treated with antimicrobials to dairy calves. Acta Vet. Scand. 55:49. https://doi.org/10.1186/ 1751-0147-55-49.

Edrington, T. S., R. L. Farrow, B. H. Carter, A. Islas, G. R. Hagevoort, T. R. Callaway, R. C. Anderson, and D. J. Nisbet. 2012 Age and diet effects on fecal populations and antibiotic resistance of a multi-drug resistant Escherichia coli in dairy calves. Agric. Food Anal. Bacteriol. 2:162-174.

EFSA Panel on Biological Hazards (BIOHAZ), A. Ricci, A. Allende, D. Bolton, M. Chemaly, R. Davies, P. S. Fernandez Escamez, R Girones, K. Koutsoumanis, R. Lindqvist, B. Norrung, L. Robertson, G. Ru, M. Sanaa, M. Simmons, P. Skandamis, E. Snary, N. Speybroeck, B. T. Kuile, J. Threlfall, H. Wahlstrom, B. Bengtsson, D. Bouchard, L. Randall, B. Tenhagen, E. Verdon, J. Wallace, R. Brozzi, B. Guerra, E. Liebana, P. Stella, and L. Herman. 2017. Risk for the development of Antimicrobial Resistance (AMR) due to feeding of calves with milk containing residues of antibiotics. EFSA J. 15. https://doi.org/10.2903/j.efsa.2017.4665.

Godden, S. M., J. P. Fetrow, J. M. Feirtag, L. R. Green, and S. J. Wells. 2005. Economic analysis of feeding pasteurized nonsaleable milk versus conventional milk replacer to dairy calves. J. Am. Vet. Med. Assoc. 226:1547-1554. 
Haas, C. N. 1994. Dose-response analysis using spreadsheets. Risk Anal. 14:1097-1100.

Haas, C. N., A. Thayyar-Madabusi, J. B. Rose, and C. P. Gerba. 2000. Development of a dose-response relationship for Escherichia coli O157:H7. Int. J. Food Microbiol. 56:153-159.

Klein-Jöbstl, D., T. Arnholdt, F. Sturmlechner, M. Iwersen, and M. Drillich. 2015. Results of an online questionnaire to survey calf management practices on dairy cattle breeding farms in Austria and to estimate differences in disease incidences depending on farm structure and management practices. Acta Vet. Scand. 57:44. https://doi.org/10.1186/s13028-015-0134-y.

Lammerding, A. M., and A. Fazil. 2000. Hazard identification and exposure assessment for microbial food safety risk assessment. Int. J. Food Microbiol. 58:147-157.

McEwen, S. A., and P. J. Fedorka-Cray. 2002. Antimicrobial use and resistance in animals. Clin. Infect. Dis. 34:S93-S106. https://doi .org $/ 10.1086 / 340246$.

Pai, C. H., J. K. Kelly, and G. L. Meyers. 1986. Experimental infection of infant rabbits with verotoxin-producing Escherichia coli. Infect. Immun. 51:16-23.

Pereira, R. V. V., T. M. A. Santos, M. L. Bicalho, L. S. Caixeta, V. S. Machado, and R. C. Bicalho. 2011. Antimicrobial resistance and prevalence of virulence factor genes in fecal Escherichia coli of Holstein calves fed milk with and without antimicrobials. J. Dairy Sci. 94:4556-4565. https://doi.org/10.3168/jds.2011-4337.

Pereira, R. V., J. D. Siler, R. C. Bicalho, and L. D. Warnick. 2014a. Multiresidue screening of milk withheld for sale at dairy farms in central New York State. J. Dairy Sci. 97:1513-1519. https://doi .org/10.3168/jds.2013-7421.

Pereira, R. V. V., J. D. Siler, R. C. Bicalho, and L. D. Warnick. 2014b. In vivo selection of resistant E. coli after ingestion of milk with added drug residues. PLoS One 9:e115223. https://doi.org/ 10.1371/journal.pone. 0115223 .

Powell, M. R., E. Ebel, W. Schlosser, M. Walderhaug, and J. Kause. 2000. Dose-response envelope for Escherichia coli O157:H7. Quant. Microbiol. 2:141-163. https://doi.org/10.1023/A:1011557402204.

Randall, L., K. Heinrich, R. Horton, L. Brunton, M. Sharman, V. Bailey-Horne, M. Sharma, I. McLaren, N. Coldham, C. Teale, and
J. Jones. 2014. Detection of antibiotic residues and association of cefquinome residues with the occurrence of Extended-Spectrum $\beta$-Lactamase (ESBL)-producing bacteria in waste milk samples from dairy farms in England and Wales in 2011. Res. Vet. Sci. 96:15-24. https://doi.org/10.1016/j.rvsc.2013.10.009.

Saini, V., J. T. McClure, D. Léger, S. Dufour, A. G. Sheldon, D. T. Scholl, and H. W. Barkema. 2012. Antimicrobial use on Canadian dairy farms. J. Dairy Sci. 95:1209-1221. https://doi.org/10.3168/ jds.2011-4527.

Simmons, R., L. Kelly, E. Snary, L. Brunton, V. Horigan, and J. Jones. 2015. A quantitative risk assessment for the selection of extended spectrum beta lactamase resistance in UK calves by feeding waste milk containing antibiotic residues. In International Symposium on Veterinary Epidemiology and Economics.

Snary, E. L. 2004. Antimicrobial resistance: A microbial risk assessment perspective. J. Antimicrob. Chemother. 53:906-917. https:// doi.org/10.1093/jac/dkh182.

Snow, L. C., R. G. Warner, T. Cheney, H. Wearing, M. Stokes, K. Harris, C. J. Teale, and N. G. Coldham. 2012. Risk factors associated with extended spectrum beta-lactamase Escherichia coli (CTX-M) on dairy farms in North West England and North Wales. Prev. Vet. Med. 106:225-234. https://doi.org/10.1016/j.prevetmed.2012 .03.009.

van den Bogaard, A. E., and E. E. Stobberingh. 2000. Epidemiology of resistance to antibiotics: Links between animals and humans. Int. J. Antimicrob. Agents 14:327-335. https://doi.org/10.1016/S0924 $-8579(00) 00145-X$.

Vasseur, E., F. Borderas, R. I. Cue, D. Lefebvre, D. Pellerin, J. Rushen. K. M. Wade, and A. M. de Passille. 2010. A survey of dairy calf management practices in Canada that affect animal welfare. J. Dairy Sci. 93: https://doi.org/10.3168/jds.2009-2429.

Wray, C., S. Furniss, and C. L. Benham. 1990. Feeding antibioticcontaminated waste milk to calves-effects on physical performance and antibiotic sensitivity of gut flora. Br. Vet. J. 146. https://doi .org/10.1016/0007-1935(90)90080-M. 